\title{
Fachbeitrag
}

Kathrin Höhner und Hans-Georg Becker

\section{Die ORCID-Integration der Technischen Universitåt Dortmund}

http://doi.org/10.1515/abitech-2019-2005

Zusammenfassung: Die Universitätsbibliothek Dortmund (UB) steht vor der Herausforderung, künftig Aussagen zum Publikationsverhalten ihrer Angehörigen treffen zu müssen. Dabei liegt die eine Schwierigkeit darin, Personen eindeutig Publikationen zuzuordnen. Zweitens müssen die Publikationsleistungen möglichst sowohl für einzelne Autoren als auch für alle Disziplinen umfassend nachgewiesen werden. Um beiden Herausforderungen zu begegnen, wurde ein Metadatenmanagementsystem entwickelt. Bereits bei der Konzeption dieses Systems wurde eine ORCID-Integration angestrebt, denn die UB sieht für beide genannten Herausforderungen die ORCID iD als Teil einer möglichen Lösung an. Die Ziele und die technische Umsetzung der Integration werden anhand der fünf Integrationsstufen, die ORCID bietet, vorgestellt.

Schlüsselwörter: Autorenidentifikation, ORCID iD, Implementierung

Abstract: TU Dortmund's university library faces two main challenges when attempting to make statements about the publication behavior of its researchers: first, associating authors to their publications unambiguously and second, obtaining an overview of publications of an individual author as well as of all disciplines. To meet both challenges a metadata management system was developed. This system was designed to be compatible with ORCID, as TU Dortmund's university library believes that the ORCID iD will be part of a potential solution to both of these challenges. The objectives and technical implementation of the integration are presented using the five levels of integration offered by ORCID.

Keywords: author identification, ORCID iD, implementation

\section{Motivation}

Die umfassende Darstellung der Publikationen von Wissenschaftlerinnen und Wissenschaftlern der eigenen
Hochschule ist ein wesentlicher Aspekt der Dokumentation der Forschungsresultate einer Hochschule. Nicht zuletzt deshalb nehmen Publikationen bei der Forschungsberichterstattung eine wichtige Rolle ein, sei es für die eigene Hochschule oder für Drittmittelgeber. Als aktuell wichtiges Beispiel sei hier der Kerndatensatz Forschung genannt. Auch für bibliometrische Analysen, die z. B. für die Evaluation von Projekten oder auch in Berufungsverfahren herangezogen werden, ist eine Übersicht über die Publikationen einer Person oder einer Einrichtung von Bedeutung.

Dafür sind die Publikationen systematisch zu erfassen: eine Kernaufgabe von Bibliotheken. Bibliotheken erschließen neben den Publikationen, die ihren Bestand ausmachen, zunehmend auch diejenigen bibliothekarisch, die von Angehörigen der eigenen Hochschule verfasst wurden. Die Präsentation erfolgt oft in Form einer Hochschulbibliographie, im Rahmen des eigenen Repositoriums oder auch als Ausgabe in Form von Publikationslisten direkt auf den Webseiten der Wissenschaftlerinnen und Wissenschaftler. Diese Dienstleistung von Universitätsbibliotheken (UB) wird gern angenommen. ${ }^{1}$

Auch die UB der Technischen Universität (TU) Dortmund sieht dies als einen wichtigen Service an. Dabei ist es ihr wichtig, verschiedene Fragestellungen beantworten zu können: Sollen Publikationen einer bestimmten Person, eines Lehrstuhls oder vielleicht eines abgegrenzten Projekts präsentiert werden? Wie hängen die Publikationen mit den einzelnen Wissenschaftlerinnen und Wissenschaftlern und diese wiederum mit den jeweiligen Einheiten der Institution oder mit Projekten zusammen?

Um Antworten auf diese Fragen zu finden, entstand bereits 2016 mit Gründung der Abteilung Informationssysteme und Datenmanagement die Idee, eine Entity Collec-

1 Vgl. hierzu: „Entsprechend ist das Interesse an der persönlichen Publikationsliste und ihrer Präsentation im Internet in den letzten Jahren gewachsen, dem besonders Einrichtungen zuständig für die Webveröffentlichung, aber auch Bibliotheken und Forschungsdezernate Rechnung tragen wollen.“ Horstmann, Wolfram, Jahn, Najko. „Persönliche Publikationslisten als hochschulweiter Dienst - Eine Bestandsaufnahme.“ Bibliothek, Forschung und Praxis 34,2 (2010): 186. doi:10.1515/bfup.2010.032. 


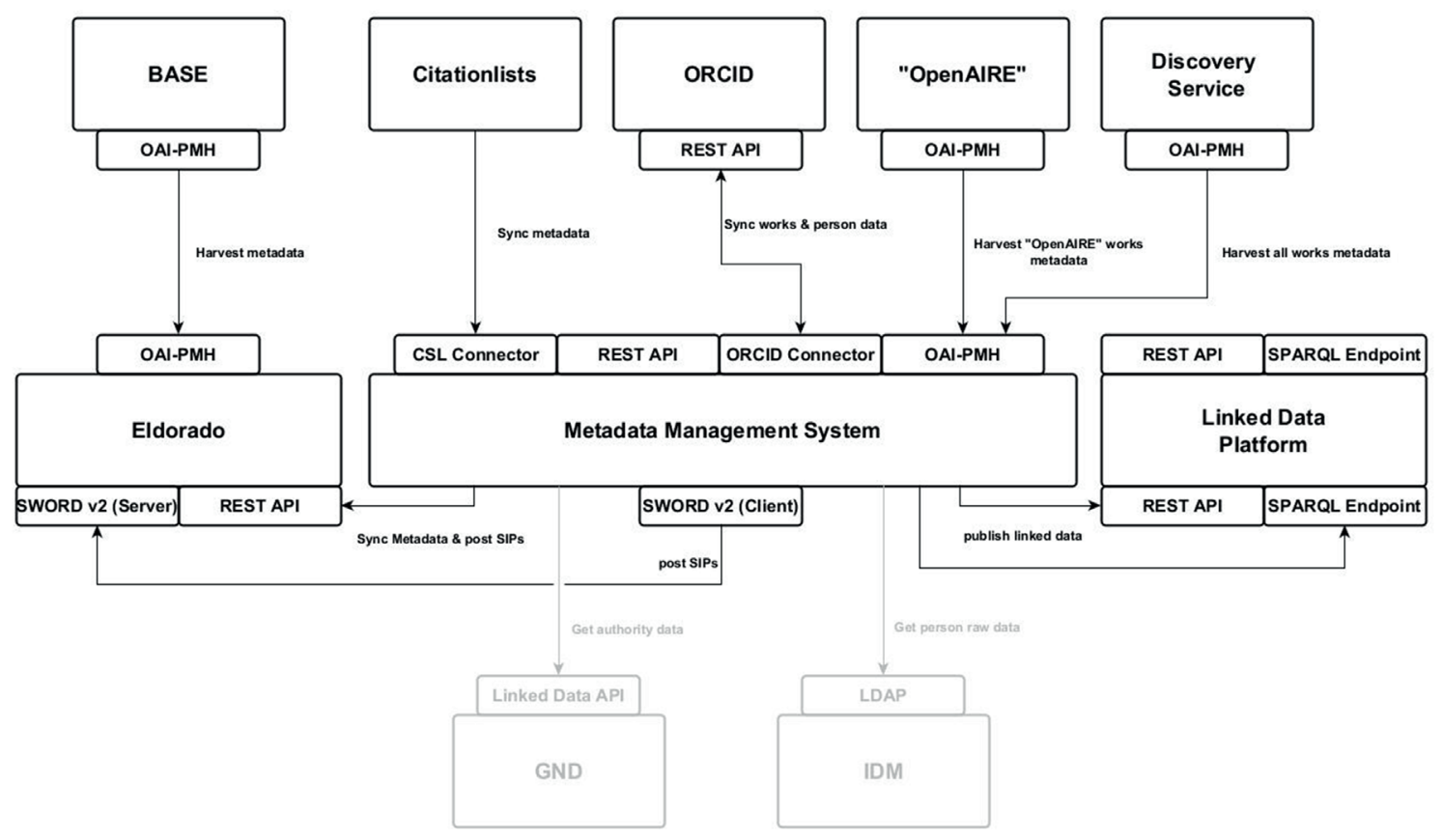

\section{(c) (i) (2)}

This work is licensed under the Creative Commons Attribution-ShareAlike 4.0 International License. To view a copy of this license, visit http://creativecommons.org/licenses/by-sa/4.0/ TU Dortmund University, University Library

Abb. 1: Datenfluss zwischen den Systemen (Quelle: Hans-Georg Becker 2017)

tion aufzubauen, in der alle Metadaten erfasst sind, die zur Darstellung der Forschungsergebnisse der TU Dortmund benötigt werden. Wie in Becker, Höhner ${ }^{2}$ beschrieben, dienen hierfür die Leitlinien der UB Dortmund für das Metadatenmanagement als Ausgangspunkt:

- Jeder Datensatz hat ein Mastersystem und ein Masterformat.

- Datensätze werden automatisch verteilt und nicht mehrfach erfasst.

- Linked Data ist ein Kernkonzept.

- Es werden so wenig Daten wie möglich und so viele wie nötig erfasst.

- Es gibt ein Repositorium für digitale Objekte.

Für den Aufbau der Entity Collection wurde in Kooperation mit der UB der Ruhr-Universität Bochum ein Metadatenmanagementsystem (MMS) entwickelt, das als Datendrehscheibe dient: Aus unterschiedlichen Quellen werden Daten eingespielt und an unterschiedliche Zielsysteme weitergegeben (vgl. Abb.1).

2 Vgl.Becker, Hans-Georg, Höhner, Kathrin. „Der Aufbau einer „Entity Collection“ der Forschungsleistung der TU Dortmund.“ doi:10.17877/ DE290R-18335 (12.04.2019).
Als Quelle dient unter anderem die ORCID-Plattform, die gleichzeitig auch Zielsystem ist. Die UB verfolgte von Anfang an den Plan, den automatisierten Datenaustausch mit ORCID in beide Richtungen zu implementieren, nämlich Daten aus ORCID zu importieren und Daten nach ORCID zu schreiben. Ein weiteres Zielsystem ist der im Rahmen der Kooperation von der UB Bochum entwickelte Publikationslistendienst, über den die Publikationen von einzelnen Wissenschaftlerinnen und Wissenschaftlern, kompletten Lehrstühlen oder Instituten auf deren Webseiten angezeigt werden.

Linked Data gemäß den Leitlinien als Kernkonzept zu betrachten bedeutet auch, an möglichst vielen Stellen zumindest aber für jede im System vorhandene Entität einen Identifikator $\mathrm{zu}$ verwenden. Hierbei ist es ein Anliegen der UB, möglichst institutionenübergreifende Identifikatoren zu verwenden. Daher setzt sie einerseits auf die GND-Nummer für Personen und Organisationseinheiten, andererseits für Personen auch auf die ORCID iD. In diesem interdisziplinären, internationalen und vor allem offenen Identifikator sieht die UB Dortmund besonderes Potential zur nachhaltigen, institutionenübergreifenden Identifikation von Autorinnen und Autoren.

Zum Konzept von ORCID gehört es, als zentrale Verteilplattform zu dienen, in die automatisch Metadaten zu 
Publikationen eingespeist werden können. ${ }^{3}$ Dies erfolgt über Schnittstellen. Um diese umfassend nutzen zu können und eine Synchronisation von ORCID mit eigenen Systemen zu ermöglichen, ist eine Mitgliedschaft bei ORCID notwendig. Die UB Dortmund ist daher an die Dekane der Fakultäten und das Rektorat herangetreten, um beide Gremien von den Vorteilen einer ORCID-Mitgliedschaft zu überzeugen. So wurde die TU Dortmund bereits Anfang 2016 - noch vor der Gründung des ORCID-Konsortiums und gemeinsam mit der Ruhr-Universität Bochum als erste Universität in Deutschland - Mitglied bei ORCID.

Um eine ORCID iD flächendeckend für Hochschulmitglieder zu etablieren, ist es allerdings notwendig, die Wissenschaftlerinnen und Wissenschaftler von den persönlichen Vorteilen des Identifikators zu überzeugen, da eine ORCID iD jede/r selbst anlegen muss; das Anlegen von ORCID iDs für andere Personen ist nicht möglich. Dies ist im Konzept von ORCID begründet, das vorsieht, dass die vollständige Datenhoheit bei dem Besitzer oder der Besitzerin der ORCID iD liegt. ${ }^{4}$ Somit erfolgt eine Disambiguierung gleichnamiger Personen direkt durch die Autoren selbst. Indem sie ihren Namen beim Einreichen einer Publikation mit ihrer ORCID iD verknüpfen, muss dies nicht mehr nachträglich erfolgen, was die Fehleranfälligkeit bei der Zuordnung von Personen zu Publikationen verringert. Dank der Verwendung der ORCID iD spielen unterschiedliche Namensvarianten keine Rolle mehr - diesen Mehrwert gilt es, den Autoren zu vermitteln.

Ein weiterer Mehrwert entsteht durch die Verknüpfung mit der UB und liegt darin, dass die Publikationsdaten durch die bibliothekarische Aufbereitung eine höhere Qualität erlangen, als es bei Importen aus anderen Quellen in den ORCID-Record der Fall ist.

Eine Verknüpfung der ORCID iD kann außerdem den Aufwand bei der Pflege persönlicher Publikationslisten verringern, indem der eigene ORCID-Record weitestgehend automatisch befüllt wird und aus dem ORCID-Record wiederum aufgrund der Verknüpfung mit der Person die Publikationsdaten in das MMS eingespielt werden können: Bei digitalen Publikationen, die in der Regel über einen Digital Object Identifier (DOI) eindeutig identifizierbar sind, findet durch die Verknüpfung von DOI und ORCID iD des Autors eine eindeutige Disambiguierung statt. Da ORCID es ermöglicht, über das sogennante Auto-Update Daten automati-

3 Vgl. DINI-Positionspapier zu ORCID: Vierkant et al. Autorenidentifikation anhand der Open Researcher and Contributor ID (ORCID) Positionspapier. DINI: Göttingen, 2018. 37. doi:10.18452/19528.

4 Vgl. Vierkant et al. 2018. 26. doi:10.18452/19528; sowie die ORCIDPrinzipien: https://orcid.org/about/what-is-orcid/principles (12.04. 2019). siert in den eigenen Record einzuspielen, ${ }^{5}$ wird Autorinnen und Autoren hier die Arbeit deutlich erleichtert. Voraussetzung ist, dass die Verwendung von ORCID iDs flächendeckend von Verlagen unterstützt wird und diese auch den DOI einer Publikation mit den ORCID iDs der Autoren oder Autorinnen verknüpfen. Bei nicht digitalen Publikationen müssen Autorinnen und Autoren die Daten weiterhin manuell pflegen - jedoch lässt sich durch die Verknüpfung der eigenen ORCID iD mit dem MMS die Datenqualität durch die bibliothekarische Aufbereitung erhöhen.

Voraussetzung für diese Erleichterungen ist, dass die Wissenschaftlerinnen und Wissenschaftler, die eine ORCID iD besitzen, der UB Dortmund entsprechende Rechte einräumen. Da die UB ihre Aktivitäten zu ORCID als Service für ihre Forschenden begreift, werden alle im MMS vorgehaltenen Publikationsdatensätze in die ORCIDRecords eingespielt, unabhängig davon, ob die jeweilige Publikation im Kontext der Tätigkeiten an der TU Dortmund entstanden ist. Ebenso werden alle Datensätze, die aus ORCID-Records in das MMS importiert werden, bibliothekarisch aufbereitet - unabhängig von deren Relevanz für die TU Dortmund.

Im Gegensatz zur Implementierung der UB Regensburg $^{6}$ sind alle aus den ORCID-Records importierten Datensätze sofort im MMS sichtbar, sofern der Status im ORCID-Record „öffentlich sichtbar“ lautet. Ebenso sind alle Datensätze im ORCID-Record sichtbar, die aus dem MMS eingespielt wurden.

Die UB selbst sieht es als sehr wichtiges Ziel an, die Vollständigkeit des Publikationsnachweises im MMS zu erhöhen. Aus dem MMS wird aktuell ein Publikationslistendienst für die Webseiten der Wissenschaftlerinnen und Wissenschaftler sowie eine Weboberfläche für eine Hochschulbibliographie gespeist. Außerdem soll das MMS als Quelle für ein Forschungsinformationssystem dienen dafür sind valide Daten notwendig, die einerseits eine eindeutige Zuordnung von Publikationen zu Personen und damit auch zu Organisationseinheiten der TU Dortmund erfordern und die andererseits so vollständig wie möglich sein sollen. Ganz pragmatisch möchte die UB die doppelte Erfassung von Publikationen vermeiden und Publikationen ihrer Forschenden nicht aktiv recherchieren müssen.

Im Folgenden wird die Implementation der Funktionalitäten von ORCID anhand der 5 Stufen, die ORCID bietet, dargestellt.

5 Für Details vgl. Vierkant et al. 2018: 32-33. doi:10.18452/19528.

6 Vgl. Deinzer, Gernot. „Von bestehenden ORCID-Integrationen in Deutschland lernen - Universität Regensburg“ (Blogbeitrag). https:// www.orcid-de.org/von-bestehenden-orcid-integrationen-in-deutsch land-lernen-universitaet-regensburg/ (12.04.2019). 


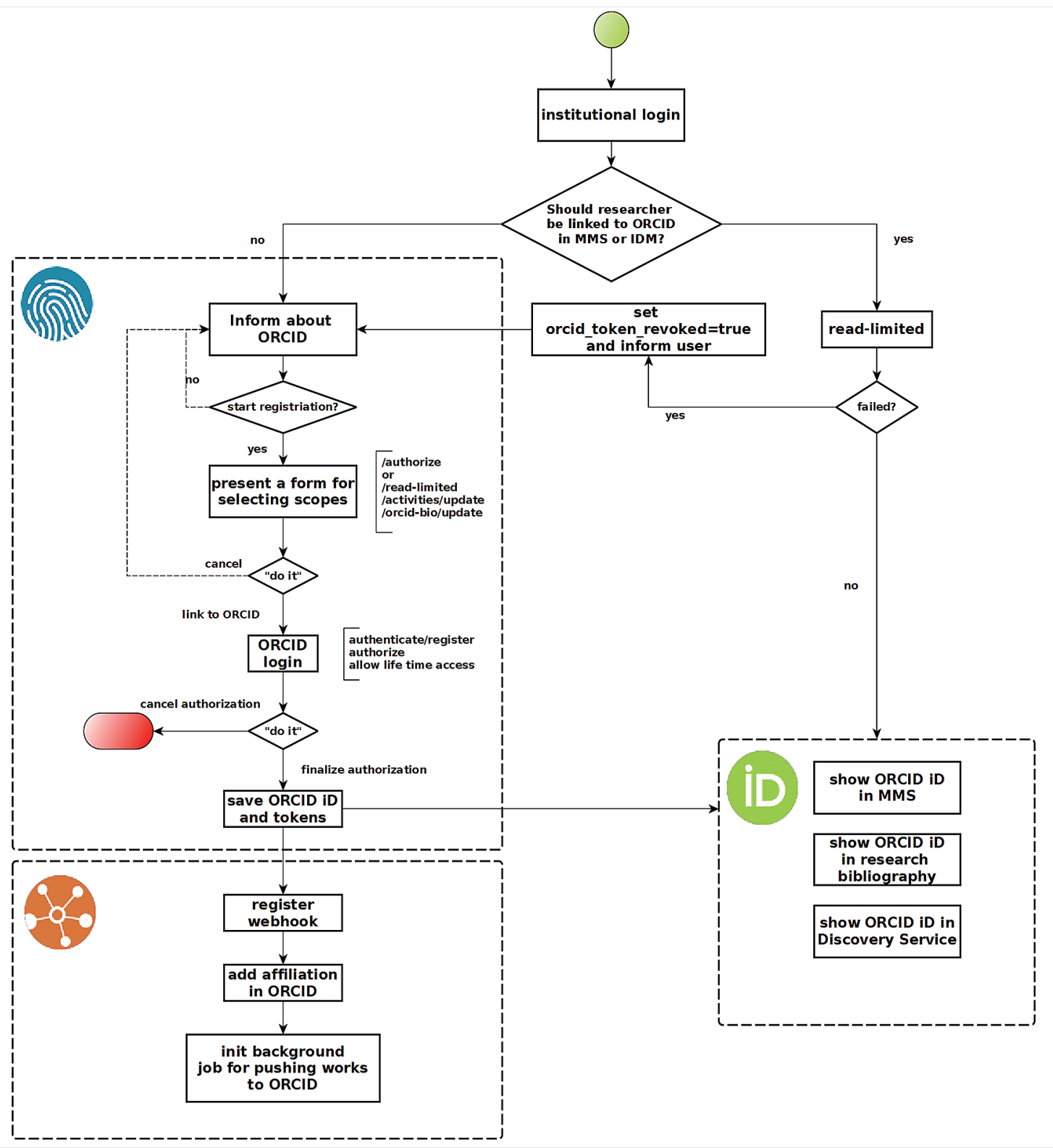

Abb. 2: Prozess zur Verknüpfung von TU-Kennung und ORCID-Record (Quelle: Hans-Georg Becker, 2018)

\subsection{Authenticate}

\subsubsection{Allgemeines}

Forschende, die eine ORCID iD besitzen, können sich mittels dieser über das MMS mit der TU Dortmund als ihrer Heimateinrichtung verknüpfen. Diese Verknüpfung wird im MMS als führendem System gespeichert und in verschiedenen Diensten angezeigt, z. B. in der Außenansicht der HSB, in Zukunft auf der digitalen Visitenkarte der Beschäftigten der TU Dortmund im Intranet sowie nach Wunsch auf den Webseiten.
Der UB war es besonders wichtig, diejenigen, die eine ORCID iD besitzen, selbst auswählen zu lassen, welche Rechte (scopes) sie der UB einräumen. Daher wurde ein vorgeschaltetes Formular entworfen und entwickelt, in dem explizit ein Haken an jedes einzuräumende Recht zu setzen ist. $^{7}$

Maximal können vier Rechte eingeräumt werden:

1. Das Recht, Daten zu lesen, die nur für Trusted Parties sichtbar sind.

7 Vgl. Höhner, Kathrin. „Rechteeinräumungen bei ORCID - ein Formulierungsvorschlag.“ (Blogbeitrag) https://www.orcid-de.org/rechte einraeumungen-bei-orcid-ein-formulierungsvorschlag/ (12.04.2019). 
2. Das Recht, diese Daten auch öffentlich in der Außensicht der Hochschulbibliographie anzuzeigen.

3. Das Recht, Daten zu Ausbildung, Beschäftigungsverhältnis, Publikationen und mehr in den ORCID-Record einzutragen.

4. Das Recht, Informationen zu weiteren Identifikatoren einzutragen. ${ }^{8}$

\subsubsection{Technische Umsetzung}

Mit dem Ziel, den institutionellen Personendatensatz eines/einer TU-Angehörigen um die verifizierte ORCID iD anzureichern, wurde im MMS für die Hochschulbibliographie die sogenannte Three-legged OAuth Authorization ${ }^{9}$ implementiert. Diese besteht aus drei Schritten:

1. Frage einen sogenannten authorization code an.

2. Wandle diesen authorization code umgehend in ein sogenanntes access token um.

3. Erhalte neben dem access token auch die autorisierte ORICD iD.

Bei der Anfrage des authorization code werden auch die Rechte mitgesendet, die diejenige Person der TU Dortmund, die die ORCID iD besitzt, für die Ausführung weiterer Funktionen rund um den ORCID-Record erteilen möchte. Diese Rechte werden - wie oben bereits beschrieben - vor dem ersten Schritt im MMS feingranular abgefragt und in eine nutzerspezifische URL zur ORCID-Plattform umgewandelt.

Für den zweiten Schritt werden neben dem authorization code auch die Client-Informationen benötigt, die eine implementierende Einrichtung bei der Registrierung der eigenen Anwendung erhält.

Die im dritten Schritt von der ORCID API erhaltenen Daten (ORCID iD, access token, refresh token und bestätigte Rechte) werden abschließend im MMS gespeichert. Diese Daten werden zum Zweck des Datenaustauschs zwischen MMS, Personendatensatz und ORCID-Record verwendet.

Zusätzlich wird ein sogenannter Webhook zur ORCID iD auf der ORCID Plattform registriert. ${ }^{10}$ Dieser sorgt dafür, dass der Service bei Änderungen am zugehörigen

8 Die konkreten Formulierungen zur Rechteeinräumung können dem Blogbeitrag entnommen werden: Höhner, Kathrin. „Rechteeinräumungen bei ORCID - ein Formulierungsvorschlag.“ https:// www.orcid-de.org/rechteeinraeumungen-bei-orcid-ein-formulie rungsvorschlag/ (12.04.2019).

9 Vgl. https://members.orcid.org/api/oauth/3legged-oauth (12.04.2019).

$10 \mathrm{Vgl}$. https://members.orcid.org/api/tutorial/webhooks (12.04.2019).
ORCID-Record darüber informiert wird. Der Webhook wird bei jeder Verknüpfung von TU-Kennung mit einem ORCIDRecord registriert, unabhängig von den erteilten Rechten. Die Benachrichtigungen von der ORCID-Plattform funktionieren auch für die öffentlichen Daten in einem ORCIDRecord, wenn kein explizites Leserecht erteilt wurde.

Im Anschluss findet eine erste Verwendung der erteilten Rechte statt. Wurde der TU Dortmund zur jeweiligen ORCID iD das Recht erteilt, Aktivitäten in den ORCIDRecord zu schreiben, so wird dort ein Eintrag über die Zugehörigkeit der jeweiligen Person zur TU Dortmund angelegt.

\subsection{Display}

Die Anzeige der ORCID iD einer Person mit dem zugehörigen Symbol ist ein wichtiger Aspekt bei der Disambiguierung von Personen. Das Symbol für ORCID signalisiert schon bei einer Kurztitelanzeige, dass der Autor oder die Autorin eindeutig zugeordnet werden kann, selbst wenn es mehrere Personen gleichen Namens unter den Autoren desselben Artikels geben sollte oder wenn mehrere Personen gleichen Namens im selben Forschungsfeld publizieren. Die UB Dortmund sieht die Anzeige der ORCID iD und des zugehörigen Symbols zudem als ein MarketingWerkzeug: Wo immer das ORCID-Symbol auftaucht, signalisiert es potentiellen Publizierenden, dass diese ID der eigenen Identifizierung dienen und die Sichtbarkeit der eigenen Publikationen erhöhen kann. Daher werden die ORCID iDs, die im MMS erfasst sind, bereits jetzt in der Hochschulbibliographie angezeigt. Zudem steht die UB in engem Austausch mit dem Rechenzentrum der TU Dortmund, das für die Anzeige der persönlichen Daten aller TU-Angehörigen auf den Webseiten und im Intranet verantwortlich ist. Auch hier wird es auf Wunsch der Forschenden möglich sein, ihre ORCID iD anzuzeigen.

\subsection{Collect and Connect}

\subsubsection{Allgemeines}

Diese beiden Funktionen zielen auf den Import von Daten aus dem ORCID-Record in das eigene System sowie das Einspielen von Daten aus eigenen Systemen in ORCIDRecords.

Für den Datenimport in das MMS spielt die Datenqualität eine wichtige Rolle. Wer eine ORCID iD besitzt, hat verschiedene Möglichkeiten, Daten in den eigenen ORCIDRecord einzupflegen: die (aufwendige) manuelle Eingabe 
oder den Import, für den verschiedene Datenbanken zur Verfügung stehen. Die Datenqualität variiert je nach Quelle für die Daten im ORCID-Record stark. Daher sind für den Datenimport in das MMS eine Qualitätssicherung sowie eine weitestgehend automatische Deduplizierung notwendig.

Auch für das Einspielen von Daten in ORCID-Records aus dem eigenen System ist es essentiell, dass zuvor eine Deduplizierung erfolgt, denn diese sollte nicht Aufgabe der ORCID-Record-Besitzenden sein. Ein weiterer wichtiger Aspekt beim Einspielen von Daten aus dem MMS ist, dass die TU Dortmund als Quelle genannt wird. Damit wird bei Publikationsdatensätzen direkt dargestellt, dass es sich um bibliothekarisch aufbereitete Datensätze handelt. Nutzt jemand seinen ORCID-Record, um eine klassische Publikationsliste als PDF-Datei herauszugeben, enthält diese Liste korrekte Literaturangaben, sofern die TU Dortmund als preferred source für die Publikationsdatensätze eingetragen wurde. Der zweite Bereich der Qualitätssicherung bezieht sich auf das Beschäftigungsverhältnis: Wurde die Information, dass eine Person an der TU Dortmund beschäftigt ist, nicht von der Person selbst, sondern von der TU Dortmund als Arbeitgeberin in den ORCID-Record eingetragen, ist dieses Beschäftigungsverhältnis verifiziert und nicht eine bloße Aussage der Person.

\subsubsection{Technische Umsetzung}

Hierbei soll es zunächst um den zweiten Teil von ORCID CONNECT gehen, der Dokumentation des Publikationsaufkommens derjenigen Person, der der ORCID-Record gehört. Das MMS bietet die Basis für Publikationslisten auf den Webseiten, und für die UB steht dieser Service im Vordergrund. Aus diesem Grund wird nicht zwischen Publikationen unterschieden, die eine Person während ihrer Zugehörigkeit zur TU Dortmund veröffentlicht hat, und solchen, die außerhalb dieses Zeitraums entstanden sind.

Der Abgleich der Publikationsdaten startet nach der Verknüpfung der Identitäten durch die Übertragung der Daten aus dem MMS in den ORCID-Record. Für jeden der Datensätze aus dem MMS wird auf dem Rückweg der Identifikator (der sogenannter Putcode) zu den UB-seitig erfolgten Angaben über die Publikation im ORCID-Record ins MMS gespeichert. Dieser Putcode wird benötigt, um die von der UB geschriebenen Daten im ORCID-Record ändern oder löschen zu können.

Werden Datensätze nach dem Erstabgleich mit dem ORCID-Record im MMS bearbeitet, startet automatisch eine Aktualisierung im ORCID-Record. Dabei kann es durchaus vorkommen, dass ein Datensatz aus dem ORCID-

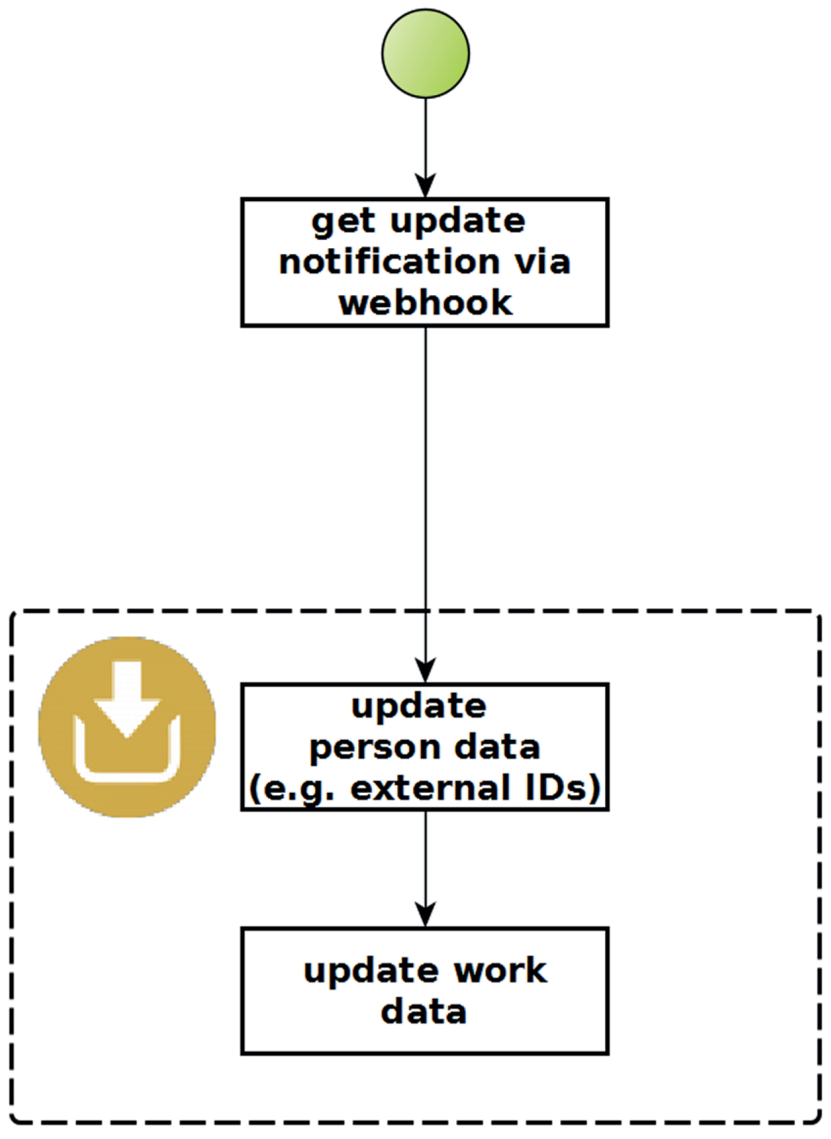

Abb. 3: Prozess zur Übernahme von Daten aus dem ORCID-Record (Quelle: Hans-Georg Becker, 2018)

Record entfernt wird, jedoch im MMS zur Hochschulbibliographie enthalten bleibt.

Durch die Registrierung des Webhooks und der darauffolgenden Speicherung der TU-Zugehörigkeit im ORCID-Record erhält der ORCID-Service innerhalb von fünf Minuten eine Mitteilung, dass es Änderungen am ORCID-Record gegeben hat. Dieses Ereignis nimmt der Service zum Anlass, die im ORCID-Record vorhandenen Publikationsdaten - und später auch Namensvarianten zur Person - zu lesen und für die Speicherung im MMS zu verarbeiten. Bei der Verarbeitung der Publikationsdaten entsteht die Herausforderung, aus einem Cluster bibliographischer Angaben im ORCID-Record eine dublettenfreie Repräsentation im MMS zu erstellen.

Eine besondere Rolle spielt daher zunächst die Prüfung, ob es zu der Publikation bereits einen Datensatz im MMS gibt. Für die Prüfung werden IDs folgender Systeme herangezogen: Digital Object Identifier (DOI), Web of Science (WOS), Scopus ID, PubMED sowie die ISBN. Liefert diese Prüfung einen bereits vorhandenen Datensatz, wird - falls nicht schon vorhanden - die ORCID iD 
zum aktuellen ORCID-Record in den MMS-Datensatz eingetragen und der Sichtbarkeitsstatus aus dem ORCIDRecord im MMS ergänzt. ${ }^{11}$ Letzterer kann dazu führen, dass ein Datensatz außerhalb des MMS (z. B. in den Publikationslisten) nicht nachgenutzt wird.

Liefert die Prüfung keinen Datensatz im MMS, so wird ein neuer Datensatz angelegt. Dabei werden aus Gründen der Datenqualität nicht unmittelbar die bibliographischen Daten aus dem ORCID-Record verwendet, sondern wenn möglich aus CrossRef oder DataCite. ${ }^{12}$

Da die Publikation im ORCID-Record aus einem Cluster bibliographischer Datensätze besteht, können verschiedene Quellsysteme Aussagen von unterschiedlicher Qualität treffen. Bei der Prüfung, ob bereits Datensätze im MMS enthalten sind, wird durch den Prozess ermittelt, ob es einen DOI gibt. Falls in den Daten des ORCID-Records kein DOI gespeichert ist, wird zunächst geprüft, ob es weitere Quellen gibt, die eine ausreichende Qualität liefern. Zuerst wird nach Daten aus Web of Science und PubMed sowie aus Scopus gesucht. Erst wenn auch hier keine Daten vorliegen, werden die Daten von ORCID verwendet. Hierbei wird dann nicht die eher rudimentäre Darstellung aus dem ORCID-Record verwendet, sondern mittels des zugehörigen Putcodes der volle Metadatensatz geholt.

\subsection{Synchronize - Technische Umsetzung}

Diese Funktion erlaubt einen kontinuierlichen Austausch zwischen dem eigenen System - an der TU Dortmund das MMS - und ORCID. Der große Vorteil liegt darin, dass keine aktive Beobachtung von ORCID-Records notwendig ist. Treten Änderungen in einem ORCID-Record auf, wird das eigene System benachrichtigt. Dabei kommt die Webhook-Technologie zum Einsatz. Für die Verwendung der Webhooks war es notwendig, dass die TU Dortmund Premium-Mitglied bei ORCID wird. Als Mitglied im Deutschland-Konsortium erfüllt sie diese Voraussetzung.

Das Prinzip hinter den Webhooks ist eine im lokalen ORCID-Service zur Verfügung gestellte Web-Schnittstelle, an die ORCID nach dortiger Registrierung eine inhaltsleere Anfrage stellt. Diese Anfrage wird mit einem bestimmten

11 Hierbei kommt es durchaus häufiger vor, dass die Publikation bereits durch die Synchronisation mit einem ORCID-Record zu einer anderen ORCID iD ins MMS gekommen ist und somit nur die Co-Autorenschaft verifiziert werden muss.

12 Der Datenimport aus CrossRef statt direkt aus dem ORCID-Record führt zu einer deutlich besseren Qualität der Daten (z.B. zu Co-Autoren und Co-Autorinnen) und wird in mehreren deutschen ORCIDImplementationen verwendet. Diese Empfehlung wird auch im Positionspapier der DINI ausgesprochen. Vierkant et al. 2018: 37.
http-Statuscode beantwortet. So „weiß“ die ORCID-Plattform, dass die Information über Änderungen am ORCIDRecord entgegengenommen wurde. Der weitere Umgang mit dieser Information ist vom eigenen System zu bestimmen; für die Integration in das MMS startet dann der im vorigen Abschnitt beschriebene Prozess.

Das Ereignis für den Datenaustausch vom MMS in die ORCID-Plattform ist das Abspeichern eines Datensatzes. Enthält dieser ORCID iDs, so werden die neuen oder geänderten Daten an die entsprechenden ORCID-Records verteilt.

\subsection{Monitoring}

Um die Funktionen rund um die Verknüpfung von ORCIDRecord und TU-Kennung sowie die Synchronisation des Publikationsaufkommens zwischen ORCID-Record und MMS besser steuern zu können, sind im ORCID-Service auch Funktionen zum Monitoring und zur Auswertung enthalten. Zum einen werden auf Anforderung Listen generiert, die erfolgreiche aber auch fehlgeschlagene Verknüpfungen von ORCID-Record und TU-Kennung anzeigen. ${ }^{13}$

Ferner werden hier die Synchronisationsjobs protokolliert. Sind diese erfolgreich abgeschlossen worden, löschen sich diese Berichte automatisch nach 24 Stunden. Bei fehlerhaften Jobs bleiben diese in dem Bericht erhalten, bis sie entweder gelöscht oder erneut gestartet werden.

\section{Allgemeines zur Implementierung}

Der in diesem Artikel beschriebene Service für die ORCIDIntegration ist vollständig in Java implementiert. Als Framework für Web-Applikationen kommt dropwizard zum Einsatz. Für die Synchronisation wird eine mittels einer Redis-Datenbank umgesetzte Queue implementiert. Eine Redis-Datenbank wird auch für die Berichte verwendet.

Die Implementierung verwendet an einigen Stellen Java Interfaces, damit lokale Anpassungen an Mappings (Crossref-MMS, DataCite-MMS, ORCID-Work-MMS) oder System-APIs (hier insbesondere das MMS) unabhängig vom Gesamt-Workflow möglich sind.

Für die Tests der ORCID-Integration wurde auf die ORCID-Sandbox zurückgegriffen. Jedoch muss dabei

13 Das Verfahren zur Verknüpfung kann beispielsweise fehlschlagen, wenn durch Netzwerkfehler im OAuth-Prozess der erhaltene $a u$ thorization code bereits wieder ungültig geworden ist. 
beachtet werden, dass diese nur eingeschränkte Funktionen bietet. So ist es hier aufgrund der Test-ORCID iDs nicht möglich, Daten aus anderen Quellen (z. B. CrossRef, Web of Science oder Scopus) zu testen. Hierfür bleibt nur der Test über die Live-ORCID-Plattform.

\section{Fazit}

Die vollständige Implementierung einer ORCID-Integration, die alle fünf beschriebenen Stufen umfasst, ist sehr komplex. Um die eigenen Anwendungsszenarien bestmöglich abzudecken, sollte daher von Beginn an genau geplant werden, wie der Datenaustausch mit ORCID erfolgen soll: Welche Systeme sollen mit ORCID kommunizieren? Soll der Austausch in beide Richtungen möglich sein? Soll er live oder in definierten Intervallen erfolgen?

Auch folgende Fragestellungen sind zu bedenken: Wie sollen die Forschenden eingebunden werden? An welcher Stelle sollen sie sich mit ihrer ORCID iD und dem eigenen System verlinken? Wie granular sollen Rechte von Forschenden eingeholt werden und wie wird für Transparenz gesorgt? Wie sind die Szenarien für die Aufhebung der Verknüpfung zwischen ORCID und dem oder den Hochschulsystemen? Wer ist an der eigenen Einrichtung für Datenhaltung verantwortlich? An der UB Dortmund wurde z. B. in der Kommunikation zwischen UB und Rechenzentrum bewusst das MMS als primäres System für die Haltung der ORCID-Daten ausgewählt.

In der Kommunikation mit den Forschenden ist es entscheidend, die Vorteile einer ORCID iD für jede einzelne
Person darzustellen. Neben der Disambiguierung ist dies die Möglichkeit, die eigene Publikationsliste im ORCIDRecord institutionenübergreifend pflegen zu können. Rückmeldungen von Forschenden an der TU Dortmund zeigen, dass die Vorzüge einer ORCID iD wahrgenommen und geschätzt werden - insbesondere in Verknüpfung mit dem Publikationslisten-Service für die eigene Webseite.

Die UB Dortmund wertet ihre Aktivitäten für eine ORCID-Integration seit 2015 als Erfolg: Wissenschaftlerinnen und Wissenschaftler schätzen die Services der UB und in der Hochschulbibliographie können Publikationen eindeutig Angehörigen der Hochschule zugeordnet werden.

\section{Autoreninformationen}

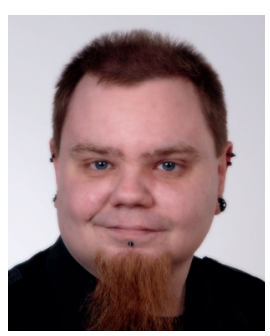

Hans-Georg Becker

Technische Universität Dortmund 44227 Dortmund hans-georg.becker@tu-dortmund.de orcid.org/0000-0003-0432-294X

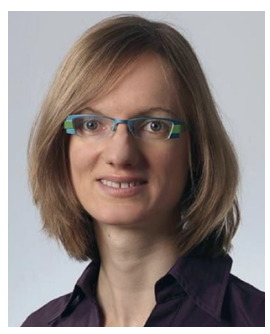

Dr. Kathrin Höhner

Technische Universität Dortmund 44227 Dortmund kathrin.hoehner@tu-dortmund.de orcid.org/0000-0002-3988-7839 